\title{
Management of childhood epiphora
}

\author{
J E Marr, A Drake-Lee, H E Willshaw
}

Br J Ophthalmol 2005;89:1 123-1126. doi: 10.1136/bjo.2005.069286

Aims: To examine the effectiveness of a management protocol for childhood epiphora using a joint ophthalmological and otolaryngological team approach.

Method: A temporally defined retrospective study of 70 children (92 eyes) undergoing surgery for persistent epiphora, despite two previous technically successful probing procedures. All the operations involved a joint approach involving a paediatric ophthalmologist and a paediatric otolaryngologist.

Results: In children with congenital nasolacrimal obstruction this joint approach yielded a $73 \%(89 \%)$ success rate, while in children with acquired nasolacrimal obstruction the success rate was $57 \%$.

Conclusions: Endonasal nasolacrimal intubation and endonasal DCR are safe and effective procedures for the management of persistent epiphora in children. They avoid the need for overnight admission and carry a minimal complication rate.

$\mathrm{E}$ piphora affects approximately $20 \%$ of neonates, but spontaneous resolution occurs in $96 \%$ of the children within the first 12 months. ${ }^{1}$ For unresolved epiphora, probing is a successful procedure ${ }^{2-4}$ with a high cure rate, although with increasing age that success rate may be reduced. ${ }^{2}$ Nasal endoscopy at the time of probing provides improved information on the site of obstruction, minimises false passages, and reduces the need for repeat procedures. ${ }^{56}$

For persistent epiphora following probing more aggressive management is required. The techniques described include balloon dacryocystoplasty, nasolacrimal intubation, standard external dacryocystorhinostomy (DCR), and endonasal DCR.

Balloon dacryocystoplasty has been somewhat disappointing. It failed to improve outcome as a primary intervention in children in one series ${ }^{7}$ and initial success rates in adults of only $57.4 \%$ (falling to $36.9 \%$ at 5 years) are reported. ${ }^{8}$ Nasolacrimal intubation, however, has previously been shown to be a successful alternative to standard external DCR after failed probing. ${ }^{10}$ Intubation may be performed "blind" or under direct intranasal endoscopic visualisation.

A recent report $^{11}$ of standard DCR performed in 121 children, with adjunct silicone intubation in $20 \%$, resulted in complete cure in $96 \%$ but only 59\% were performed as day cases. Complications included postoperative cellulitis in three cases and one suture granuloma.

In adults endonasal DCR compares favourably with the conventional external approach in terms of symptomatic relief, with the benefit of a lower complication rate $\mathrm{e}^{12-14}$ and the greater use of day case, rather than inpatient beds. In children, despite the technical challenges posed by small anatomical dimensions, endonasal endoscopic DCR has been reported to offer an alternative to standard external DCR in two series, ${ }^{15}{ }^{16}$ though unfavourable outcomes were reported in a third. ${ }^{17}$

\section{AIM}

We present the results of our collaborative ophthalmological and ENT endonasal procedures for persistent epiphora in children and provide a rationalised plan for the management of childhood epiphora.

\section{Study design}

This is a retrospective case series of 92 eyes of 70 children undergoing surgery, for persistent congenital or acquired nasolacrimal system obstruction. Children were admitted between January 1991 and September 2003. All the children had previously undergone two technically successful probing procedures, but had persistent symptoms. A successful outcome was indicated by symptomatic relief.

The investigation was undertaken before ethical approval for retrospective studies was a requirement in our institution. All data were anonymised.

\section{Subjects \\ Congenital nasolacrimal duct obstruction}

Sixty four children ( 85 eyes) had been symptomatic since birth. The mean age at presentation to the tertiary referral centre was 36 months (range 5 months to 11 years). Seventeen of these 64 children had other associated abnormalities (table 1).

\section{Acquired nasolacrimal duct obstruction}

Six children (seven eyes) presented with acquired epiphora. The mean age at presentation was 7 years (range $1-11$ years). In three eyes the epiphora occurred after acute dacryocystitis in previously asymptomatic children.

\section{METHODS}

\section{Endonasal intubation}

Following induction of anaesthesia, a cotton wool applicator soaked in $4 \%$ cocaine is placed in the nose under the inferior turbinate. After 5 minutes the applicator is removed and the inferior turbinate infractured with a Hills elevator. The upper and lower puncta are dilated and the lacrimal system probed using an "O" or "OO" gauge Bowman probe. Following successful probing, under direct visualisation with a nasal endoscope, "Concept" tubing (Meditronic, Jacksonville, FL, USA) is introduced through each punctum in turn and retrieved from the nose, once again under direct visualisation. A Watzke sleeve (Labtician, Oakville, Ontario, Canada) is placed over the cut end of the tubes. The inferior turbinate is then repositioned. The tubes are left in situ as shown in table 2, after which they are removed under general anaesthesia.

\section{Endonasal DCR}

Following induction of anaesthesia, two cotton wool applicators soaked in $4 \%$ cocaine are placed in the nose adjacent to the anterior end of the middle turbinate and middle meatus. After 5 minutes the applicators are removed and an injection

Abbreviations: DCR, dacryocystorhinostomy. 
Table 1 Associated pathology

\begin{tabular}{lc}
\hline Abnormality & Number of children (number of eyes) \\
\hline Choanal atresia & $1(1)$ \\
Craniosynostosis & $2(2)$ \\
Down's syndrome & $6(9)$ \\
Midfacial dysmorphism and/or & $6(8)$ \\
cleft & $1(1)$ \\
Russell-Silver syndrome & $1(1)$ \\
Unknown dysmorphic syndrome & \\
\hline
\end{tabular}

of 2\% lignocaine and adrenaline 1:80 000 is made submucosally. A fibreoptic light pipe is introduced through the lower canaliculus to identify the lacrimal fossa for the endonasal surgeon. If the nose is large enough a $0^{\circ} 2 \mathrm{~mm}$ diameter Hopkins rod is inserted into the nose and used for the procedure. If the nasal aperture is too small, an illuminated Killians endoscope is used. The mucosa over the floor of the lacrimal fossa is elevated and then the bone is drilled with an angled drill to create an ostium of approximately $1.5 \mathrm{~cm}$ diameter. The lacrimal sac is opened under direct vision. Tubes are then inserted as above and the inferior turbinate relocated. The tubes are left in situ for 6 weeks and then removed as described previously.

\section{RESULTS}

Congenital masolacrimal duct obstruction alone

Forty seven children (63 eyes) had congenital nasolacrimal duct obstruction with no other associations. The procedures undertaken, complications, and outcomes are summarised in table 2. A successful outcome was obtained in 46/63 (73\%) eyes.

The outcome in 10 eyes was not known-this was largely because those children had been referred from outside units who then undertook postoperative follow up. The children were subsequently discharged from those units on the understanding they would return if they remained symptomatic. If one were to assume that failure to return indicates successful outcome then the overall success rate in this group would rise to $89 \%$.

\section{Congenital nasolacrimal duct obstruction and associated problems}

Seventeen children (22 eyes) had congenital nasolacrimal duct obstruction plus associated conditions. A successful outcome was obtained in 18/22 eyes (82\%).

\section{Acquired nasolacrimal duct obstruction}

Six children (seven eyes) presented with acquired symptoms. The mean age at presentation was 7 years (range $1-11$ years). In three eyes the epiphora occurred after acute dacryocystitis in previously asymptomatic children. A successful outcome was obtained in four children (four eyes). One child presented at the age of 5 months with bilateral darcryocystitis following an episode of conjunctivitis having been previously symptomatic. She was lost to follow up after the first postoperative visit.

\section{Procedures}

The outcomes of all the procedures in the different patient groups are shown in figure 1.

\section{Intubations}

Seventy eight intubations were undertaken and the result was identified in 70 . The success rate was 53/70 (76\%).

\section{Endonasal DCRs}

Three endonasal DCRs were performed as primary procedures, one following a previously failed DCR, and 12 following unsuccessful intubations. The overall success rate was $14 / 16(87 \%)$.

\section{External DCRs}

Primary, four (two successes; outcome unknown in two); following intubation, three (one success (33\%)); following endonasal DCR, three (two successes $(66 \%)$ ); overall success, $5 / 8(63 \%)$

\section{COMPLICATIONS}

An intraoperative complication occurred in only one child. This was a burn from the shaft of the drill to the nostril and it did not affect the surgical outcome. A mild postoperative epistaxis occurred in one child.

Tubes were pulled out by the patient or fell out early in 11 cases and only one subsequently failed. A tube prolapse occurred in one child necessitating removal of the tubes at one month and this case subsequently failed.

A nasal discharge in one child necessitated early tube removal and once again this did not affect the success of the procedure. Asymptomatic cheesewiring occurred in three children who were symptomatically cured. Cheesewiring occurred in one girl with mid-facial dysmorphism who was not cured, but the cheesewiring was not thought to be a significant factor in the procedures failure. A punctal granuloma occurred in one case and this case subsequently failed.

Table 2 Surgical intervention and outcome-congenital nasolacrimal duct obstruction

\begin{tabular}{|c|c|c|c|c|}
\hline Procedure sequence & Outcome & $\mathbf{n}$ & Tubes out (mean) (range) & Complications \\
\hline \multirow[t]{4}{*}{ Intubation alone } & Cure & 38 & $\begin{array}{l}5 \text { months (1 day- } \\
10 \text { months) }\end{array}$ & $\begin{array}{l}\text { Symptomatic cheesewiring in } \\
\text { three cases }\end{array}$ \\
\hline & Not known & 8 & Not known & None \\
\hline & Fail & 4 & 3 months (1-3 months) & None \\
\hline & Fail & 1 & 3 days & Punctal granuloma \\
\hline \multirow[t]{3}{*}{ Intubation + endo DCR } & Cure & 5 & Not recorded & None \\
\hline & Cure & 1 & 6 months & $\begin{array}{l}\text { Intra-op nostril burn + late } \\
\text { cheesewire }\end{array}$ \\
\hline & Fail & 1 & 1 month & Tube prolapse \\
\hline Intubation + endo DCR + & Cure & 1 & 3 days & \\
\hline external DCR & Cure & 1 & 6 months & Cheesewire \\
\hline Intubation + external DCR & Cure & 1 & Not recorded & None \\
\hline External DCR & Cure & 1 & Not recorded & None \\
\hline $\begin{array}{l}\text { Endo DCR (previous failed } \\
\text { external DCR at other centre) }\end{array}$ & Cure & 1 & 6 months & None \\
\hline
\end{tabular}




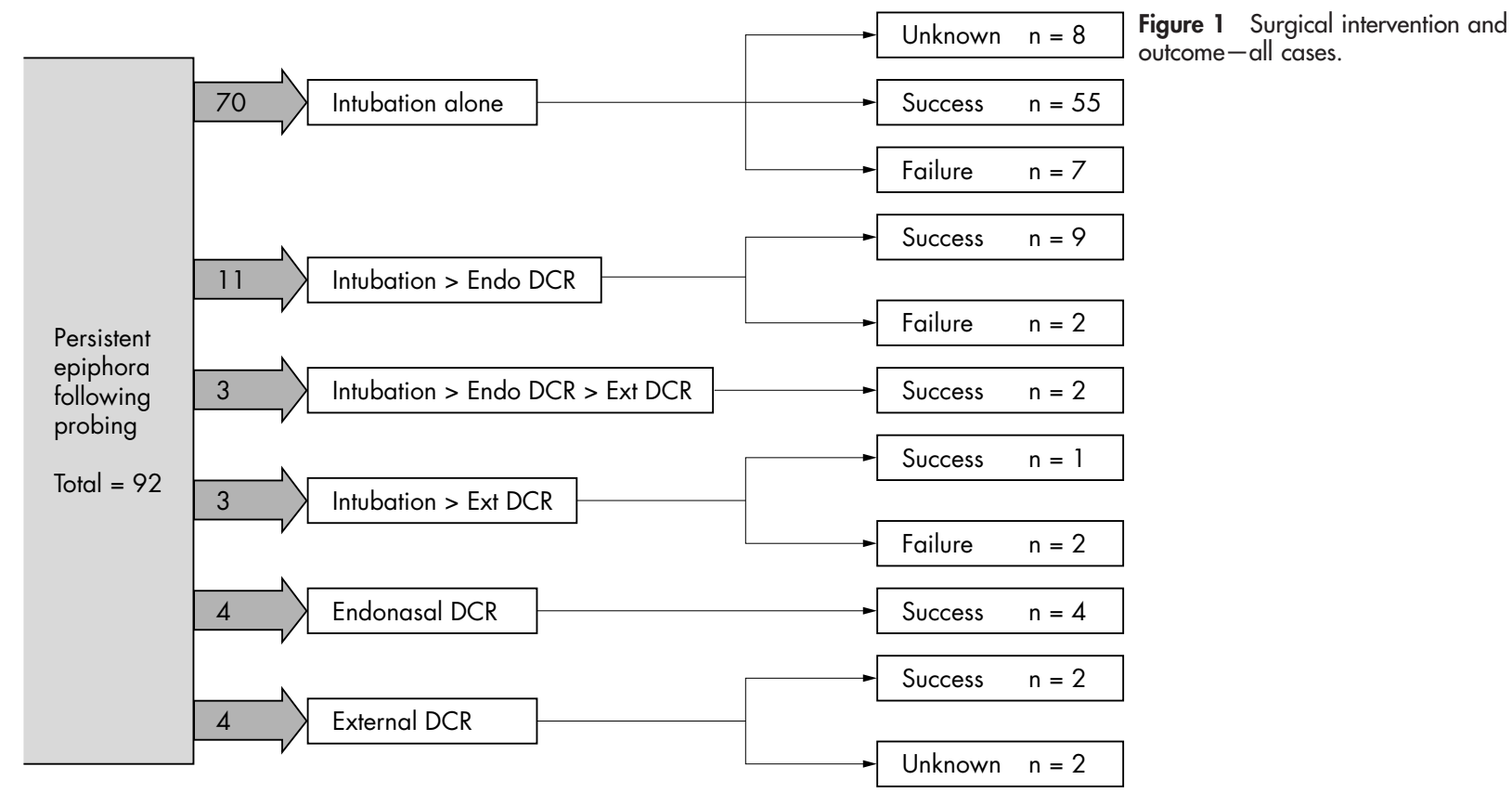

\section{DISCUSSION}

This is a retrospective assessment of the outcome of surgery in 92 children with epiphora who had previously failed two probing procedures. All the surgery was performed endonasally, by a joint ophthalmology/ENT team, and the large majority of the children were followed up by that team to determine symptomatic relief and, where possible, lacrimal system patency. A single clinician skilled in endonasal surgery could undertake the various procedures; however, in our experience the technical difficulties associated with endonasal surgery in children make a team approach preferable.

The great majority of the children had congenital nasolacrimal obstruction, and in that group at least $73 \%$ and possibly $89 \%$ were cured. The presence of associated systemic problems did not significantly reduce the success rate, despite the fact that six children (nine eyes) had Down's syndrome which is known to carry a poorer prognosis for lacrimal surgery. The much reduced success rate in children with acquired nasolacrimal obstruction is predictable, but even in this group the results are sufficiently good to warrant an attempt at endonasal surgery before proceeding to "ab externo" surgery.

Nasolacrimal intubation performed as a joint procedure is quick and relatively complication free. The correct tensioning of the tubes is necessary to avoid cheesewiring through the puncta (when the tubes are too tight) or tube prolapsed from the puncta (when the tubes were too loose) requires experience, but cheesewiring when it occurred did not adversely affect the cure rate. Tube prolapse was distressing for the family, but did not cause the child any great discomfort and does not (in our experience) cause any corneal epithelial damage. However, because it often necessitated early tube removal it could lead to surgical failure, as happened in one of our cases.

This raises the question of the optimum duration for the tubes remaining in situ. In a previous report ${ }^{9}$ it was recommended that the tubes remain in place for 6 months. However, subsequent studies have suggested that this is unnecessarily long and our current practice is to remove the tubes after 6 weeks.

Endonasal DCR, though technically difficult in small children, offers a number of advantages over the ab externo approach. It avoids the need for a skin incision and consequent scarring, it enables accurate creation of a drainage ostium even when the lacrimal sac is small and scarred, and it is accompanied by much more limited peroperative bleeding. Using a joint ophthalmology/ENT approach, we were able to cure $87 \%$ of those children requiring DCR. All these children were treated as day cases, and the only complication was a small burn to the ala caused by a drill tip (the use of a drill to create the ostium is not always necessary, but a significant number of these children have thickened bone in the floor of the lacrimal fossa, and a drill or laser should always be available).

This contrasts with a previous report $^{11}$ in which an extremely impressive 96\% cure rate was achieved with ab externo DCR but where 52 of 127 admissions required overnight stay, and three children developed orbital cellulitis and required systemic antibiotics.

\section{CONCLUSIONS}

Endonasal intubation and endonasal DCR are effective procedures, and play an important role in the management of childhood epiphora. Early tube removal did not adversely affect the outcome in the majority of cases in which it occurred, and removal at 6 weeks should now be regarded as the norm. Complication rates were low and $100 \%$ day case rates were achieved for the endonasal procedures.

\section{Authors' affiliations}

J E Marr, H E Willshaw, Department of Paediatric Ophthalmology, Children's Hospital, Birmingham, UK

A Drake-Lee, Deparment of Paediatric Otolaryngology, Children's Hospital, Birmingham, UK

Competing interests: none declared

Correspondence to: $\mathrm{Mr} \mathrm{H} \mathrm{E} \mathrm{Willshaw,} \mathrm{Department} \mathrm{of} \mathrm{Paediatric}$ Ophthalmology, Children's Hospital, Steelhouse Lane, Birmingham B4 6NH, UK; harry.willshaw@bch.nhs.uk

Accepted for publication 23 April 2005 


\section{REFERENCES}

1 Machete CJ, Yound JDH. Epiphora during the first year of life. Eye 1991;5:596-600.

2 Robb RM. Success rates of nasolacrimal duct probing at time intervals after year of age. Ophthalmology 1998;105:1309-10.

3 Mannor GE, Rose GE, Frimpong-Ansah K, et al. Factors affecting the succes of nasolacrimal duct probing for congenital nasolacrimal duct obstruction. Am J Ohthalmol 1999;127:616-17.

4 Sturrock SM, MacEwen CJ, Young JD Long term results after probing for congenital nasolacrimal duct obstruction. Br J Ophthalmol 1994;78:892-4.

5 MacEwen CJ, Young JD, Barras CW, et al. Value of nasal endoscopy in the diagnosis and management of children with congenital epiphora. Br J Ophthalmol 2001;85:314-18.

6 Ram B, Barras CW, White PS, et al. The technique of nasendoscopy in the evaluation of nasolacrimal obstruction in children. Rhinology 2000;38:83-6.

7 Gunton KB, Chung CW, Schnall BM, et al. Comparison of balloon dacryocystoplasty to probing as the primary treatment of congenital nasolacrimal duct obstruction. J AAPOS 2001;5:139-42.

8 Lee DH, Song HY, Ahn H, et al. Balloon dacryocystoplasty: results and factors influencing outcome in 350 patients. J Vasc Interv Radiol 2001;12:500-6.
9 Aggarwal RK, Misson GP, Donaldson I, et al. The role of nasolacrimal intubation in the management of childhood epiphora. Eye 1993;7:760-2

10 Beigi B, O'Keefe M. Results of Crawford tube intubation in children. Acto Ophthalmologica 1993:71:405-7.

11 Barnes EA, Abou-Rayyah Y, Rose GE. Paediatric dacryocystorhinostomy for nasolacrimal duct obstruction. Ophthalmology 2001;108:1562-4.

12 Cokes Y, Evereklioglu C, Er H. Comparative external versus endoscopic dacryocystorhinostomy:results in 115 patients (130 eyes). Otolaryngology Head Neck Surg 2000;123:488-91.

13 Eloy P, Bertrand B, Martinez M, et al. Endonasal dacryocystorhinostomy: indications, techniques and results. Rhinology 1995;33:229-33.

14 Dzhambazov KB. Endonasal microscopic dacryocystorhinostomy: results and advantages. Folia Medica (Plovdiv) 2000:42:51-6.

15 Cunningham MJ, Woog JJ. Endonasal endoscopic dacryocystorhinostomy in children. Arch Otolaryngol Head Neck Surg 1998; 124:328-33.

16 Vanderveen DK, Jones DT, Tan H, et al. Endoscopic dacryocystorhinostomy in children. J AAPOS 2001:5:133.

17 Doyle A, Russell J, O'Keefe M. Paediatric laser DCR. Acta Ophthalmologica Scandinavic, 78:204-5. 